\title{
Differential Insertion Depths of Filiform Needle, Concept and Application
}

\author{
Lei Li*, Clara W. C. Chan, Kwai Ching Lo \\ School of Chinese Medicine, The University of Hong Kong, Hong Kong, China \\ Email:"llie@hku.hk
}

Received 25 September 2015; accepted 26 December 2015; published 29 December 2015

Copyright (C) 2015 by authors and Scientific Research Publishing Inc.

This work is licensed under the Creative Commons Attribution International License (CC BY). http://creativecommons.org/licenses/by/4.0/

(c) (i) Open Access

\section{Abstract}

In this paper, the background, evolution, basic meaning, clinical application and the detail operating procedures of the differential insertion depth in filiform needle acupuncture were discussed based on the classical expositions of the Yellow Emperor's Canon of Medicine. It is believed that the differential insertion depth reflects the basic idea of expelling the evil Qi from the body in the application of traditional acupuncture. Since the site of evil invasion has different shades, the position of evil Qi and correct differentiation has become the operation key points of needle insertion. Apart from this, the Yellow Emperor's Canon of Medicine has further associated the clinical application of filiform needle insertion depth with the seasonal change of Yin and Yang, the body built of the patients, the nature of the diseases, the heat or cold pathogenic factors of the illness, the excess and deficiency of the patient, and the reinforcing and reducing function of acupuncture. These elaborations have greatly enriched the basic content of acupuncture and laid a systematic theoretical foundation of filiform needle operation. The differential insertion depth in acupuncture has its specific meaning, the emphasis of insertion depth of filiform needle with its differentiated clinical implication exemplifies the perceptual thinking features of traditional acupuncture and typical reveals the uniqueness of Chinese civilization.

\section{Keywords}

Yellow Emperor's Canon of Medicine, Traditional Acupuncture, Differential Insertion Depths of Filiform Needle

\section{Introduction}

Differential Insertion depths are a basic integral part of traditional acupuncture. It describes the appropriate in-

${ }^{*}$ Corresponding author. 
sertion depths of filiform needle and the related clinical implications.

Acupuncture is the main instrument used by Yellow Emperor's Canon of Medicine to describe and explain the theories and therapeutic principles of Traditional Chinese Medicine. Yellow Emperor's Canon of Medicine has established the basic theories, and thoroughly discussed the principles and treatment methods of acupuncture and moxibustion. It is therefore recognized as the most influential classic doctrine in the development history of acupuncture. Based on the relevant content of Yellow Emperor's Canon of Medicine, this paper has explored and discussed the background, evolution, basic meaning, and clinical implications of applying differential insertion depths in filiform needle treatment, with the aim of providing a framework of reference for today's scientific and clinical researches.

\section{The Origin of "Differential Insertion Depths" of Filiform Needle}

The application of differential insertion depths in filiform needle treatment has reflected the fundamental belief of Ancient Chinese that acupuncture was used to expel evil Qi from the body.

Ancient Chinese believed that disease was primarily the consequence of spirits haunting, or ancestral punishment or taboo violation. Hence, the disease was located exactly where the haunted site was and expelling spirits from the haunted location in the body was the principal guideline to heal.

During the early stage of developing the theory of acupuncture, the basic concept was that evil spirits invaded the body and hid in the acupoints, or "painful points", which formed a relatively superficial structure. They were visible to the naked eye, close to the blood vessels and easy to locate at the body surface. Stone wedges, horns, needles, moxibustion and massage were the major tools and techniques, which the ancient Chinese applied to dispel the evil spirits at the acupoints [1].

In the era when Yellow Emperor's Canon of Medicine took shape, which is commonly believed to be between the Warring States period (475-221BC) and the early Han dynasty (206BC-220AD), with the invention of Nine Needles and filiform needles becoming particularly popular in clinical application, the common understanding of the nature and structure of acupoints changed, and the concepts of acupoints and meridians began to merge. Acupoints were perceived as the locations where the Qi of the human body gathered and dispersed, and also the stage where the righteous Qi and evil Qi competed against each other. The concept of the causes of diseases also evolved: instead of evil spirits haunting the human body, diseases were attributed to various pathogenic reasons. However, using acupuncture to dispel evil Qi has remained the key concept throughout Yellow Emperor's Canon of Medicine.

When evil Qi invades the human body, the affected locations are naturally at different depths. Hence, the key purpose of acupuncture treatment is to reach the exact location, specifically the exact depth of the location where the evil Qi resides so as to effectively dispel the evil Qi from the body.

\section{The Basic Meaning of Differential Insertion Depths}

The concept of differential insertion depths of filiform needle, as expounded in Yellow Emperor's Canon of Medicine, placed special emphasis on determining the insertion depths to take account of 1) the nature of the evil Qi resided in the acupoints and 2) the depth where the evil Qi was resided.

As mentioned in Spiritual Pivot: Nine Needles and Twelve Yuan:

"When Qi has invaded the Channels, Xieqi (Evil-Qi) is in the upper, Zhuoqi (Turbid-Qi) is in the middle and Qingqi (Lucid-Qi) is in the lower. So needling the Acupoints located in the depressions eliminate Xieqi (Evil-Qi), needling the Zhongmai eliminates Zhuoqi (Turbid-Qi) and deep needling when the disease is superficial leads to internal invasion of Xieqi (Evil-Qi) and worsening of the disease.” [2]

It clearly explained that the pathogenic Qi resided in the acupoint could be differentiated into Xieqi, Zhuoqi and Qingqi, and these pathogenic Qi resided at different depths. Hence, the inserted needle needs to reach the appropriate depth in order to dispel the pathogenic Qi. If the needle is inserted deeper than is appropriate, it could lead the pathogenic Qi to go deeper and aggravate the disease.

A similar concept was also discussed in Plain Conversation: Discussion on Regulation of Channels:

"If the disease is in the Channel, it can be treated by regulating blood; if the disease is in the blood, it can be treated by regulating the Collaterals; if the disease is in the Qi Phase, it can be treated by regulating the Wei (Defensive-phase); if the disease is in the muscles, it can be treated by regulating the muscles; if the diseases is in the sinews, it can be treated by regulating the sinews; if the disease is in the bone, it can be treated by regu- 
lating the bone." [3]

This is to say that pathological changes caused by different diseases will take place in specific parts of the body. The filiform needle must reach the specific locations where the pathological changes take place in order to heal. i.e. If the changes take place in the blood, the blood needs to be recuperated; if the changes affect the Qi, the Qi needs to be regulated; if it happens to the sinew or bone, the respective affected parts should be strengthened accordingly.

It was mentioned in Spiritual pivot: Application of Needles:

"The key of needling lies in the reasonable application of the needles. The nine kinds of needles have different usages. They are either long, or short, or large, or small. They are used for different purposes. Wrong use of them cannot cure diseases. Deep insertion of needles in treating superficial diseases will damage muscles and cause skin abscess. Shallow insertion of the needle in treating deeply located diseases will, instead of expelling pathogenic factors, cause superlative ulcer. The use of large needle in treating mild diseases will excessively reduce Qi and worsen the disease. The use of small needle in treating severe disease cannot expel pathogenic factors and cure the disease."

It was also mentioned in Plain Conversation: Discussion on the essential of Acupuncture:

'Huandi said: 'I'd like to know the essentials of needling.' Qibo answered: 'Diseases are either external or internal and needling can be either shallow or deep. To treat diseases, needles should be inserted into the required depth, neither too deep nor too shallow. If it is inserted too deep, it will cause internal damage; if it is inserted too shallow, it will cause external stagnation, giving rise to the invasion of Xieqi (Evil-Qi). Hence improper depth of needling brings about great disaster that affects the Five Zang-Organs, and leads to serious diseases. That is why it is said that diseases are located sometimes in the body hair and Rouli (Muscular-Interstices), sometimes in the skin, sometimes in the muscles, sometimes in the Channels, sometimes in the sinews, sometimes in the bones and sometimes in the bone marrow.

So in needling the surface of body and Rouli, care should be taken not to impair the skin. Impairment of the skin disturbs the lung, leading to Wennue (Warm-Malaria) in autumn with symptoms of chills and aversion to cold. In needling the skin, care should be taken not to impair muscles. Impairment of muscles will disturb the spleen, leading to abdominal distension and fullness and anorexia in the last eighteen days in each season, amounting to seventy-two days altogether. In needling muscles, care should be taken not to impair the Channels. Impairment of the Channels disturbs the heart, leading to heart pain in summer. In needling the Channels, care should be taken not to impair the sinews. Impairment of the sinews disturbs the liver, leading to febrile diseases and flaccidity of sinews in spring. In needling the sinews, care should be taken not to impair the bones. Impairment of the bones disturbs the kidney, leading to abnormal distention and lumbago in winter. In needling the bones, care should be taken not to impair the marrow. Impairment of the marrow reduces the marrow, leading to weakness of the legs and lassitude of the body. So the patient does not like to move."

Both paragraphs have clearly explained why there should be different depths of needle insertion. The differentiation should be made according to the depth and precise location of the targeted organ or tissue. If the pathological changes happened in the inner area, then the needle should penetrate deeply; if the pathological changes took place near the body surface, the needle should be inserted shallowly. Should the acupuncturist be unable to insert the needle to the appropriate depth, like inserting the needle to a shallow area when the pathological change happened in the inner body, or inserting the needle deeper than the area of pathological change, adverse consequences will occur.

In Spiritual Pivot: Application of Needles, an acupuncture methodology named "triple needling to induce Guqi” was discussed. It divided needle insertion depths into three levels, namely the intradermal, subcutaneous and the muscle levels, and mentioned that the depth of needle puncture should be decided according to the depth of the exact locations of the evil Qi and righteous Qi. This is probably the most classic discussion on differential insertion depths in The Yellow Emperor's Canon of Medicine.

As mentioned in Spiritual pivot: Application of Needles:

"The needling method known as triple needling for inducing Guqi (Food-Qi) means to puncture the skin first to dissipate Yangxie (Yang-Evil); then puncture a little deeper into the muscles without reaching the muscular interstice in order to remove Yinxie (Yin-Evil); and finally deepen the needle into the muscular interstice to conduct Guqi (Food-Qi). That is why the book titled Needling Methods says, 'Shallow needling is used at first to expel Xieqi (Evil-Qi) and promote blood circulation; deep needling is then used to discharge Xie (Evil) from the Yin phase; and extreme deep needling finally is used to conduct Guqi (Food-Qi).' The reason is just what is 
mentioned above."

This is to say that the needle should first be inserted into the shallow part of the selected acupoint to dispel the evil Qi of yang phase which resided there; then the needle should be inserted deeper to dispel the evil Qi of yin phase. After all the evil Qi is expelled, the needle could then be inserted even deeper to conduct the righteous Qi. By doing so, the disease could be healed and the Qi and blood of the local area could be regulated and normality could be regained.

The purpose of applying differential insertion depths in acupuncture is to dispel the evil Qi in the local area, this is the fundamental meaning of utilizing differential insertion depths in acupuncture as mentioned in Yellow Emperor's Canon of Medicine.

\section{The Interpretation and Expansion of the Concept of Differential Insertion Depths of Filiform Needles in Yellow Emperor's Canon of Medicine}

Although the fundamental consideration of utilizing differential insertion depths in acupuncture is to dispel the localized evil Qi, Yellow Emperor's Canon of Medicine has further expanded its implication in clinical application. Apart from the exact location where the evil Qi resides, Yellow Emperor's Canon of Medicine suggested that in deciding the insertion depths of the needle, reference should also be made to the yin and yang balance of the four seasons, the body built of the patients, the nature of the diseases, the heat or cold pathogenic factors of the illness, the excess and deficiency of the patient, and the reinforcing and reducing function of acupuncture. The concept of differential insertion depths of the filiform needle is thus related to all aspects of the clinical practice of acupuncture.

It was mentioned in Spiritual Pivot: Beginning and End:

"The invasions of pathogenic factors into the body vary in depth in different seasons. In spring, pathogenic factors tend to attack hairs; in summer, pathogenic factors tend to attack the skin; in autumn, pathogenic factors tend to attack muscular interstices; and in winter, pathogenic factors tend to attack tendons and bones. Thus the treatment of these diseases with needling should be done according to changes of seasons. So to use needling therapy to treat obese patients, the methods used in autumn and winter should be used; to use needling therapy to treat thin patients, the methods used in spring and summer should be used."

This clearly explained that evil Qi resides in different parts of the human body as the seasons change. Therefore, when to conduct acupuncture treatment, the insertion depth of the filiform needle should be decided according to the seasons. Shallow needling should be applied in spring, summer and deep needling should be applied in fall, winter. When treating obese patients, deep needling like those to be used in fall and winter seasons should be applied, and when treating thin patients, shallow needling like those to be used in spring and summer should be applied.

A similar concept was mentioned in Spiritual pivot: Yin and Yang, Lucidity and Turbidity:

"So in needling the Yin Channels, the needles should be inserted deeply and retained for a longer period of time; in needling the Yang Channels, the needles should be inserted shallowly and with-drawn quickly."

This further explained that in treating diseases occurred in the Yin Phase, the needle should be inserted deeply and retained longer, whereas in treating diseases occurred in the Yang Phase, the needle should be inserted shallowly and withdrawn quickly.

As mentioned in Spiritual pivot: Beginning and Ending:

"Pain is usually caused by accumulation of pathogenic cold and therefore belongs to Yin Syndrome and pain which is deeply rooted and cannot be felt by pressure with a fingers also pertains to Yin Syndrome. These kinds of Yin Syndrome can be treated by deep needling. Itching is a problem pertaining to Yang and should be treated by shallow needling."

This suggested that all pain syndromes including those where the hurting locations could not be identified are diseases of Yin and need to be dealt with by inserting the needle deeply. All diseases manifest as itching belong to Yang, and need to be dealt with by inserting the needle shallowly.

As discussed in Spiritual pivot: Abnormality, Normality, Obesity and Emaciation:

"People who are in the supreme of life and whose blood and Qi are sufficient, skin is solid, when attacked by Xie (Evil), can be treated by deep insertion and longer retention of the needles. This is the way to deal with heavy people. Their shoulders and armpit are usually broad, their muscles are thin and skin is thick and black, their lips are plump and thick, their blood is blackish and turbid, the Qi in their body is unsmooth and slow in 
flowing. This kind of people tends to keep forging ahead and is also generous to others. To use acupuncture therapy to treat this kind of people, the needles should be inserted deeply and retained for a longer period of time. At the same time the frequency of needling can be increased.

Huangdi said, 'How to deal with thin people then?' Qibo said, 'Thin people are characterized by thin skin, light color emaciated muscles, thin lips, low voice, clear blood and swift Qi. So the Qi tends to be exhausted and the blood tends to be damaged. To use acupuncture to treat this kind of people, the needle should be inserted shallowly and swiftly.'

Huangdi said, 'How to deal with average people?' Qibo said, 'They can be treated by regulating respectively according to the degree of whiteness and blackness of the skin. Those who are honest and sincere with regular features and whose blood and Qi are in harmony can be treated by needling without violating the common practice.'

Huangdi said, 'How to deal with strong people?' Qibo said, 'Strong people are usually characterized by solid bones, chubby and strong muscles and relaxed and nimble joints. If they move slowly, it is usually due to un-smooth flow of Qi and turbidity of blood. To treat this kind of people with needling, the needle should be inserted deeply and retained for a longer period of time. And the frequency of needling also should be increased. If they move swiftly, it indicates that the Qi is slippery and the blood is clear. To treat this kind of people with needling, the needle should be inserted shallowly and withdrawn swiftly.'

Huangdi asked, 'how to deal with babies?' Qibo said, 'Babies are characterized by thin muscles and skin, shortness of Qi and weakness of blood. So to treat babies with needling therapy, the filiform needles should be used. The needles should be inserted shallowly and withdrawn quickly. Such a treatment is performed once the other day."”

This illustrated that needle should be inserted deeply for fat person and shallowly for the thin person and babies. The depth of needle insertion should be decided according to the patient's overall conditions when acupuncture is applied to a normal person.

As discussed in Spiritual pivot: Root and Knot:

"The general rule is that to treat those whose Qi is swift, the needle should be withdrawn quickly; to treat those whose Qi is unsmooth, the needle should be withdrawn slowly; to treat those whose Qi flows rapidly, the needle should be small and inserted shallowly; to treat those whose Qi is unsmooth, the needle should be big and inserted deeply. The deeply inserted needle should be retained in the acupoint while the shallowly inserted needle should be withdrawn quickly."

This explained that shallow needling technique should be applied if the Qi and blood of the patient flows swiftly and smoothly, while deep needling technique should be applied if the Qi and blood of the patient flows unsmoothly or became stagnated.

As mentioned in Spiritual pivot: Symptoms of Zangfu-Organs due to Attack of Pathogenic Factors:

"All kinds of rapid pulse indicate cold; all kinds of slow pulse indicate heat; ... So the needling technique for rapid pulse and the related disease should be deep and the needle should be retained in the selected acupoint for a longer period of time. The needling technique for slow pulse and the related disease should be shallow and the needle should be withdrawn immediately after insertion in order to remove heat."

This explained that the patient's disease is normally hot in nature when his pulse is slow, then, shallow needling technique should be applied, on the other hand, the patient's disease is normally cold in nature when his pulse is rapid, then, deep needling technique should be applied.

As mentioned in Spiritual pivot: Beginning and Ending:

"Forceful pulse indicates excess of pathogenic factors which should be treated by deep needling in order to expel evil Qi. Weak pulse indicates deficiency of Healthy-Qi which should be treated by shallow needling in order to prevent Jingqi (Essence-Qi) from leaking, invigorate the pulse and drain Xieqi (Evil-Qi).”

This elucidates that the insertion depth of the needle should be deep when the patient's pulse is strong and forceful while the insertion depth of the needle should be shallow when the patient's pulse is weak.

As mentioned in Spiritual pivot: Channels and Collecterals:

"The heat ones can be treated by swift needling techniques; the cold ones can be treated by retaining the needles in the acupoints."

It illustrated that diseases with heat syndromes should be treated by shallow needling technique and the needle should be withdrawn quickly, whereas diseases with cold syndromes should be treated by deep needling technique with the needle retained in the acupoint for a considerably longer period of time. 
As mentioned in Spiritual pivot: Beginning and Ending:

"Reinforcing and reducing techniques should be performed according to the conditions of pulse. If the pulse is forceful, the needles should be inserted deeply. After the withdrawal of the needle, the needled acupoint is not pressed immediately in order to drain Xieqi (Evil-Qi). If the pulse is weak, the needles should be inserted shallowly in order to invigorate the pulse. After the withdrawal of the needle, the acupoints should be pressed immediately to prevent invasion of Xieqi (Evil-Qi). ”

This clearly explained that the insertion depth of the needle should be shallow and the acupoint should be pressed immediately after the withdrawal of needle when the goal of the acupuncture was to reinforce the Righteous Qi. Whereas, the insertion depth of the needle should be deep and the acupoint should not be pressed immediately if the goal of the acupuncture was to dispel or reduce the Evil Qi.

The differential insertion depths of filiform needles in Yellow Emperor's Canon of Medicine are hereby classified in Table 1.

The appropriate insertion depths of filiform needle therefore become an important part of clinical acupuncture practice. In general, if the pathological changes in the interior, the needle should be penetrated deeply; if the lesion occurs near the surface, the needle should be inserted shallowly. For example, in treating lumbago due to kidney deficiency, it should be deep needling; in treating fever caused by exogenous pathogens, it should be shallow needling; wry eye and mouth should be shallow needling, abdominal distension and diarrhea should be deeply needling.

The extensive use of differential insertion depths has vastly enriched the essential content of the application technique of filiform needle in acupuncture, which in turn, laid a solid foundation for the theoretical and systematic approach of the filiform needle application technique.

\section{The Combined Needling Techniques and Differential Insertion Depth(s) of Filiform Needles in Yellow Emperor's Canon of Medicine}

Various types of combined needling techniques were discussed in Yellow Emperor's Canon of Medicine, like the five needling methods which correspond to the five Zang-Organs; nine ways of needling to deal with the nine kinds of pathological changes and the twelve methods of needling to correspond to the twelve meridians discussed in Spiritual Pivot: Application of Needles.

Most of these combined needling techniques have incorporated the concept of differential needle insertion depths such as the five needling methods corresponding to the five Zang-Organs:

"There are five needling methods to correspond to the five Zang-Organs. The first one is called 'Banci' (half needling) which means to insert needle superficially and withdraw the needle quickly without damaging the muscles. This way of needling, just like pulling body hair, is used to expel evil Qi from the skin. The second one is called 'Baowenci' (leopard spot needling) which means to insert the needles into the left, the right, the anterior and posterior regions around the affected part. In such a treatment the needles must be inserted into the

Table 1. The differential insertion depths of filiform needles in Yellow Emperor's Canon of Medicine.

\begin{tabular}{ccc}
\hline Classification & Shallow needling & Deep needling \\
\hline Position of evil Qi & Superficial body & Deep body \\
Seasons & Spring and summer & Autumn and winter \\
Body built of patients & Thin & Fat \\
Qi and blood running & Fast and smoothly & Slowly and unsmoothly \\
Pulse manifestation & Slow and weak & Rapid and strong \\
Nature of diseases & Yang syndrome & Yin syndrome \\
Pain and itch & Itch & Pain \\
Heat or cold of illness & Heat syndrome & Cold syndrome \\
Excess and deficiency of patients & Deficiency syndrome & Excess syndrome \\
Reinforcing and reducing manipulations & Reinforcing method & Reducing method \\
\hline
\end{tabular}


Channels for the purpose of bloodletting. Such a treatment corresponds to the heart. The third one is call 'Guanci' (joint needling) which means to directly needle the joints in the four limbs and the distal part of the tendon to treat Jinbi (Bi-syndrome of tendons). Cares should be taken to avoid bleeding. Such a treatment corresponds to the liver. It is also called 'Yuanci' and 'Qici'. The fourth one is called 'Heguci' (Tri-directional needling) which appearing like the talon of a chicken, means to insert the needle deep into the muscular interstices to treat Jibi (Bi-Syndrome of muscle). Such a treatment corresponds to the spleen. The fifth one is called 'Shuci' (transmitted needling) which means to insert and withdraw the needle perpendicularly. The needle is inserted deep onto the bone to treat Gubi (Bi-Syndrome of bone). Such a treatment corresponds to the kidney."

When utilizing needling techniques which correspond to the five body parts, the acupuncturist will divide the acupoints into five levels according to the respective positions of skin, muscle, vein, tendon and bone, and insert the needle to the appropriate depth to treat the pathological changes in the lung, heart, liver, spleen and kidney systems respectively.

Again take for example the "Jingci", "Luoci", "Fenci" and "Maoci" of the nine ways of needle application to treat various pathological changes.

"Jingci which means to puncture that part of the large Channel that connects with the Collateral."

"Luoci which means to puncture the small Collaterals to let out the blood."

"Fenci which means to puncture the part between muscles."

"Maoci which means to puncture the skin beneath which there is floating Bi-Syndrome."

The needle needs to reach the Channels in "Jingci" and thus it is a deep needling technique. The needle needs to reach the Collaterals in "Luoci", thus it is a shallow needling technique. The needle is targeted to reach the muscle layer in "Fenci", thus it is a deep needling technique and the needle is targeted to reach the skin in "Maoci" and thus, it is a shallow needling technique.

Similarly, "Huici", "Qici”, "Yangci”, "Zhizhenci”, "Shuci”, "Duanci”, "Fuci” and "Zanci” of the twelve Channels corresponding needling technique also incorporate the concept of differential insertion depths:

"Huici (extended needling), which means to insert the needles around the spasm of muscles. The needles are lifted and thrusted forwards or backwards to relax muscles and treat Jinbi (Bi-Syndrome of tendons)."

"Qici (triple needling), which means to insert one needle perpendicularly into the affected part and two more beside to treat cold disease with small scale and deep penetration of Qi (pathogenic factor)."

"Yangci (scattered needling), which means to insert one needle first into the centre of the affected part and then four more around. The needles are inserted shallowly to treat cold disease that involves a large region."

"Zhizhenci (direct needling), which means to pinch the muscle and insert the needle into it. This way of needling is used to treat cold disease with shallow location."

"Shuci (transmitting needling), which means to insert and withdraw the needle perpendicularly. Usually fewer Acupoints are selected and punctured deeply. This way of needling is used to treat the disease marked by superabundance of Qi and severity of heat."

"Duanci (gradual needling), which used to treat Gubi (Bi-Syndrome of the bone), means to insert the needle deep into the bone, slightly shake the needle and deepen the insertion till the needle reaches the bone. Then the needle is manipulated upward and downward to rub the bone."

"Fuci (floating needling), which means to insert the needle superficially beside the affected part in order to treat cold spasm of muscle."

"Zanci (supplemental needling), which means to insert and withdraw the needle perpendicularly. Usually several needles are inserted superficially to let out blood. Such a way of needling is used to treat carbuncle and swellings."

"Huici" is to insert the needle to the tendon; "Qici" targets to treat cold disease with small scale and deep penetration of Qi; "Shuci" is to insert the needle deeply in a few acupoints; "Duanci" is to insert the needle deeply to the bone to treat Bi-Syndrome of bone. These are all considered to be Deep Needling techniques. On the other hand, "Fuci" is to insert the needle to the superficial layer of the muscle; "Zanci" inserts the needles superficially to release blood; "Yangci" is to insert the needle shallowly to release the evil Qi; and "Zhizhenci" is to pinch the skin and insert the needle horizontally. These are all considered to be Shallow Needling techniques.

When the concept of differential insertion depths is applied to the combined needling techniques, special emphasis is put on the nature of diseases; the exact location of the pathological changes; the severity of the diseases and the changes of application techniques. It revealed, from a different angle, the high flexibility and wide clinical application of the filiform needle treatment and the fundamental purpose of acupuncture as dispelling the 
evil Qi from the body. Thus, it is an integral part of the fundamental knowledge of filiform needle operation.

\section{Conclusion}

Filiform needle treatment in traditional acupuncture emphasizes the clinical implications of differential insertion depths. It is a classic example of intuitive direct visualization, which is a unique characteristic of Chinese civilization. Based on the conditions of the diseases, different insertion depths are applied, and it has greatly broadened the spectrum of application techniques of acupuncture using filiform needles. The comprehensive discussion of differential insertion depths in Yellow Emperor's Canon of Medicine has greatly expanded the clinical application of filiform needle treatment. The concept of differential insertion depths has not only enriched the content of filiform needle treatment, but also well integrated with the reinforcing and reducing needling technique to lay the systematic theoretical foundation of filiform needle treatment. Various well-known needle manipulation techniques have developed in the later years, like "Shaoshanhuo (Heat-producing Needing)", "Toutianliang (Cool-producing Needing)”, “Qinglongbaiwei (Green Dragon Shaking Tail)”, "Baihuyaotou (White Tiger Shaking Head)", "Cangguitanxue (Dark-green Tortoise Seeking for Cave)" and "Chifengyingyuan (Red Phenix Meeting Resource)", etc. are all the interpretation and development of the concept of differential insertion depths in the Yellow Emperor's Canon of Medicine.

\section{References}

[1] Li, L., Yau, T. and Yau, C.H. (2012) What is the Origin of Acupoint. Journal of Acupuncture and Tuina Science, 10, 125-127. http://dx.doi.org/10.1007/s11726-012-0587-8

[2] Anonymous (1956) The Spiritual Pivot (Copied Print). People’s Health Publishing House, Beijing.

[3] Anonymous (1956) Yellow Emperor’s Internal Classics: Plain Conversation (Copied Print). People’s Health Publishing House, Beijing. 